\title{
METODO_SCGE UNA METODOLOGÍA BASADA EN SERVICIOS COMPUTACIONALES PARA EL DESARROLLO DE GOBIERNO ELECTRÓNICO
}

\author{
Marcelo Castro ${ }^{1}$, Claudia Fabiana Pons ${ }^{2}$ and Rocío Andrea Rodríguez ${ }^{3}$ \\ ${ }^{1}$ Facultad de Ingeniería, Universidad Nacional de Jujuy \\ Ítalo Palanca $N^{\circ} 10$, San Salvador de Jujuy, Jujuy, Argentina \\ ${ }^{2}$ Laboratorio de Investigación y formación en Informática Avanzada Universidad Nacional de La Plata \\ Calle 50 esq.115 1er.Piso, La Plata, Argentina \\ ${ }^{3}$ Departamento de Ingeniería e Investigaciones Tecnológicas \\ Universidad Nacional de la Matanza, Buenos Aires, Argentina
}

\begin{abstract}
RESUMEN
En este trabajo se presenta la validación de las dos primeras etapas de una metodología para sistematizar y estandarizar los procesos de Gobierno Electrónico en la gestión pública, a través de servicios computacionales (metodo_SCGE). La metodología considera tanto el software, como el hardware y las comunicaciones, el análisis de estos componentes permite generar un modelo integral basado en servicios computacionales para gobierno electrónico (SCGE). El artículo contiene una breve introducción al concepto de servicios computacionales en el ámbito de gobierno electrónico y una pequeña descripción de la metodología. Al final se presenta un resumen sobre la validación de dos etapas, en un servicio específico perteneciente a un organismo gubernamental.
\end{abstract}

\section{PALABRAS CLAVE}

Servicios Computacionales, Metodologías de e_gov, Servicios al Ciudadano

\section{INTRODUCCION}

El objetivo del trabajo es presentar metodo_SCGE incluyendo la validación de la primera y segunda etapa en un caso de estudio específico. Es importante señalar que la propuesta de desarrollo de la metodo_SCGE fue presentado en el año 2018 en eventos científicos de la disciplina (Cas-18). Para el desarrollo de metodo_SGE se tuvieron en cuenta metodologías de análisis y diseño de software existentes, que van desde modelos de desarrollo estructurado con metodologías orientadas al flujo de datos, hasta modelos de desarrollo orientado a objetos como el Lenguaje Unificado de Modelado (Boo-04) o más recientemente las Arquitecturas orientadas a Servicios o SOA (Erl-08). La metodología desarrollada propone integrar en un SCGE, tanto los aspectos relacionados al software, como a la estimación de los recursos computacionales. El proyecto Access-eGov ha desarrollado herramientas de software que permiten la integración de servicios utilizando tecnologías semánticas (Hre-11). La Arquitectura Dirigida por Modelos o MDA (Mel-04) realiza aportes significativos en el desarrollo de software. Existen aspectos que podrían complementarse a las citadas metodologías como son la estimación de los recursos computacionales y por otra parte se hace necesaria una metodología específica para el diseño de soluciones de gobierno electrónico, esos aspectos son cubiertos por metodo_SCGE y representan la diferencia con los trabajos señalados precedentemente. 


\section{METODO_SGE: UNA METODOLOGÍA BASADA EN SCGE}

La metodología metodo_SCGE se encuentra basada en servicios computacionales,un SCGE es una unidad de servicio que se pone a disposición de una solución de gobierno electrónico, la cual puede estar compuesta por varios SCGE y estos a su vez conformar varias soluciones de gobierno electrónico. A continuación se describirán los tres componentes que conforman a un SCGE.

\subsection{Componentes}

Un SCGE está compuesto por:

- Una parte dirigida al análisis, diseño, prueba e implementación del software de gobierno electrónico; denominada Componente Software. Contiene las funcionalidades específicas del dominio de e_gov, también contiene la descripción del sistema operativo, el lenguaje o herramienta de desarrollo utilizada, el sistema Gestor de Base de Datos y la arquitectura de software implementada.

- Un elemento relacionado con la estimación de los recursos computacionales necesarios para soportar el software de gobierno electrónico, denominado Componente Hardware y Conectividad. Este componente se refiere a los recursos de hardware y conectividad asociados a la solución diseñada.

Como puede apreciarse en la figura 1 un SCGE podría graficarse de la siguiente manera:

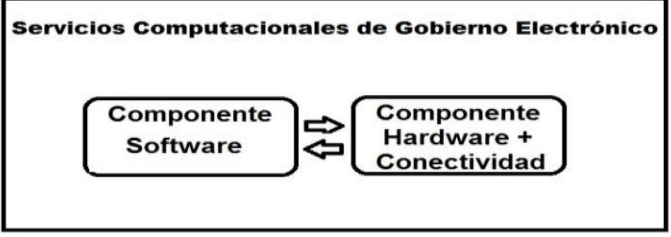

Figura 1. Componentes de un Servicio computacional de Gobierno Electrónico

\subsubsection{Componente Software}

Este componente es de suma importancia debido a que contiene las funcionalidades concretamente definidas para el SCGE. Estas funcionalidades se encuentran directamente relacionadas con las especificadas en el trabajo "Análisis de las propiedades y atributos propios de sitios de gobierno electrónico" (Cas-09) y representan el núcleo del SCGE. En este componente se deben especificar el sistema operativo nativo sobre el cual se encuentra soportado el servicio, el lenguaje de programación utilizado para generar el código fuente o generado por un entorno integrado de desarrollo (IDE), o una herramienta de ayuda para desarrollar aplicaciones (CASE) utilizada para el desarrollo del servicio, además el sistema gestor de Base de Datos que se utilizará y la Arquitectura de Software que soportará el SCGE

\subsubsection{Componente Hardware}

Aquí se deberá definir el equipamiento que soportará el SCGE diseñado. En primer lugarresulta indispensable consignar la unidad central de procesamiento (CPU) que se utilizará, como así también el tipo y la capacidad de la memoria RAM requerida, el tipo y tamaño del soporte de almacenamiento magnético que resulte necesario. Por último es necesario establecer el tipo y características de las interfaces que se usarán. Para poder definir convenientemente este componente resultará adecuado realizar una estimación de los recursos computacionales necesarios, tales como tipo y capacidad de procesamiento y almacenamiento. En la Figura 3 se puede visualizar la estructura del componente descripto.

\subsubsection{Componente Conectividad}

En este componente se define la manera en que el SCGE se encuentra conectado con otros sistemas y servicios computacionales, se deberá definir el tipo de enlace físico a utilizar, la velocidad de conexión, el protocolo de comunicación y el tipo de red. En la figura 4 se puede observar la estructura del componente de conectividad. En la Figura 2 puede observarse la estructura de los componentes Software, Hardware y Conectividad. 


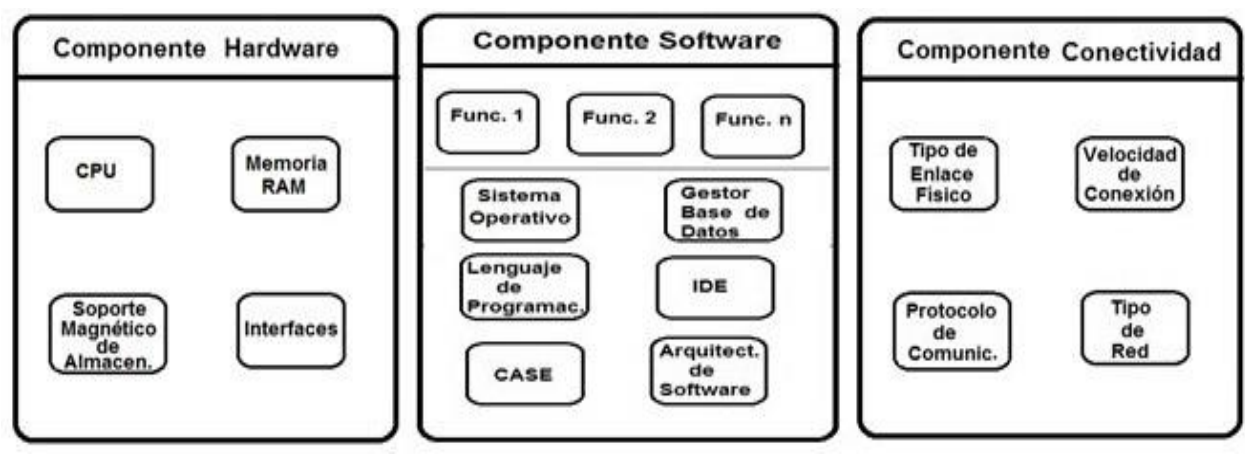

Figura 2. Estructura de los componentes Software, Hardware y Conectividad

\subsection{Características de la Metodología Basada en SCGE (metodo_SCGE)}

Se trata de una metodología iterativa e incremental de desarrollo basado en SCGE. Al igual que las metodologías ágiles tradicionales para el desarrollo de software, metodo_SCGE realiza incrementos e iteraciones para agregar no sólo funcionalidades, sino también características de software, hardware y conectividad para el SCGE que se está desarrollando.

\subsection{Etapas de Metodo_SCGE}

La metodología metodo_SCGE posee cuatro etapas, las cuales se describen a continuación:

Etapa 1- Seleccionar características de Componentes SCGE de versión a liberar: en esta etapa se seleccionarán las características de software, hardware y conectividad que se desean incorporar en la nueva versión que se liberará.

Etapa 2- Desglosar características de cada componente: en esta etapa se deben determinar claramente las características que se desean desarrollar para cada componente.

Etapa 3- Desarrollar, utilizar, integrar y probar cada característica por componente: aquí se realizará el desarrollo, la integración y las pruebas de cada característica correspondiente a cada uno de los componentes de un SCGE.

Etapa 4- Liberar y evaluar versión del SCGE: durante esta etapa se procederá a la liberación y evaluación de la versión desarrollada para el SCGE.

En la figura 3, se puede observar un gráfico de las etapas de metodo_SCGE.

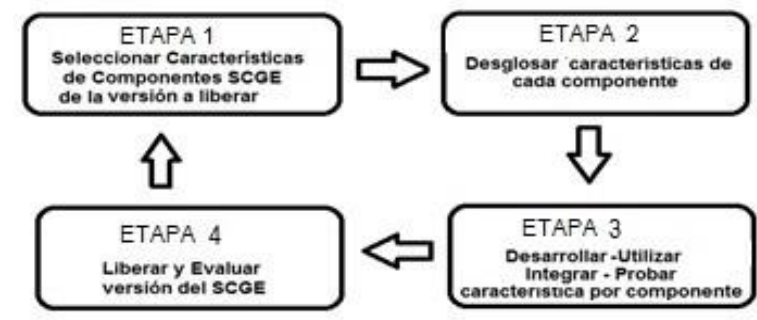

Figura 3. Etapas de metodo_SCGE

\subsection{Actividades de Metodo_SCGE}

En la tabla 1, se detallan las actividades a llevar a cabo en cada una de las etapas de la metodología propuesta: 
Tabla1. Actividades por etapas de metodo_SCGE

\begin{tabular}{|l|l|l|l|}
\hline \multicolumn{1}{|c|}{ Etapa } & $\begin{array}{c}\text { Actividad/Comp } \\
\text { onente }\end{array}$ & \multicolumn{1}{|c|}{ Etapa } & Actividad/Componente \\
\hline $\begin{array}{l}\text { I- Seleccionar características de } \\
\text { Componentes SCGE de la } \\
\text { versión a liberar }\end{array}$ & $\begin{array}{l}\text { I.1Seleccionar } \\
\text { característica de } \\
\text { Software (CS) }\end{array}$ & $\begin{array}{l}\text { III-Desarrollar, utilizar, integrar } \\
\text { y probar cada característica por } \\
\text { componente }\end{array}$ & III.1- Desarrollar, utilizar, integrar y probar CS \\
\hline & $\begin{array}{l}\text { I.2-Seleccionar } \\
\text { característica de } \\
\text { Hardware (CH) }\end{array}$ & & III.2-Desarrollar, utilizar, integrar y probar CH \\
& $\begin{array}{l}\text { I.3-Seleccionar } \\
\text { característica de } \\
\text { Conectividad (CC) }\end{array}$ & & III.3-Desarrollar, utilizar, integrar y probar CC \\
\hline $\begin{array}{l}\text { II-Desglosar características de } \\
\text { cada componente }\end{array}$ & $\begin{array}{l}\text { II.1-Desglosar } \\
\text { característica CS }\end{array}$ & $\begin{array}{l}\text { IV-Liberar y evaluar versión del } \\
\text { SCGE }\end{array}$ & $\begin{array}{l}\text { IV.1-Implementar y evaluar la versión liberada del } \\
\text { SCGE, para los tres componentes }\end{array}$ \\
\hline & $\begin{array}{l}\text { II.2-Desglosar } \\
\text { característica CH }\end{array}$ & & \\
\hline & $\begin{array}{l}\text { II.3-Desglosar } \\
\text { característica CC }\end{array}$ & & \\
\hline
\end{tabular}

\subsection{Validación de Metodo_SCGE Aplicándola a un Caso de Estudio en un Organismo Gubernamental}

La metodología metodo_SCCGE está siendo validada en la Dirección Provincial de Rentas de la Provincia de Jujuy, la cual depende de la Secretaría de Ingresos Públicos perteneciente al Ministerio de Hacienda. Para la aplicación de metodo_SCGE se utilizaron algunos de los servicios a brindar al ciudadano y que se encuentran detallados en el trabajo (Cas-09) para la característica "Funcionalidad" de los sitios de GE, atributo "Poder Ejecutivo" y sub-atributo "Servicios públicos, finanzas y producción”. En la tabla 2 se detalla la validación de las dos primeras etapas, correspondiente al componente software (CS).

Tabla 2. Validación de las dos primeras etapas correspondiente al componente software (CS)

\begin{tabular}{|c|c|c|}
\hline Etapas & $\begin{array}{c}\text { Actividades/Co } \\
\text { mponentes }\end{array}$ & Especificaciones \\
\hline $\begin{array}{l}\text { I- Seleccionar } \\
\text { características } \\
\text { de } \\
\text { Componentes } \\
\text { SCGE de la } \\
\text { versión a } \\
\text { liberar } \\
\text { II-Desglosar } \\
\text { características } \\
\text { de cada } \\
\text { componente }\end{array}$ & $\begin{array}{l}\text { I.1Seleccionar } \\
\text { característica de } \\
\text { Software (CS) }\end{array}$ & 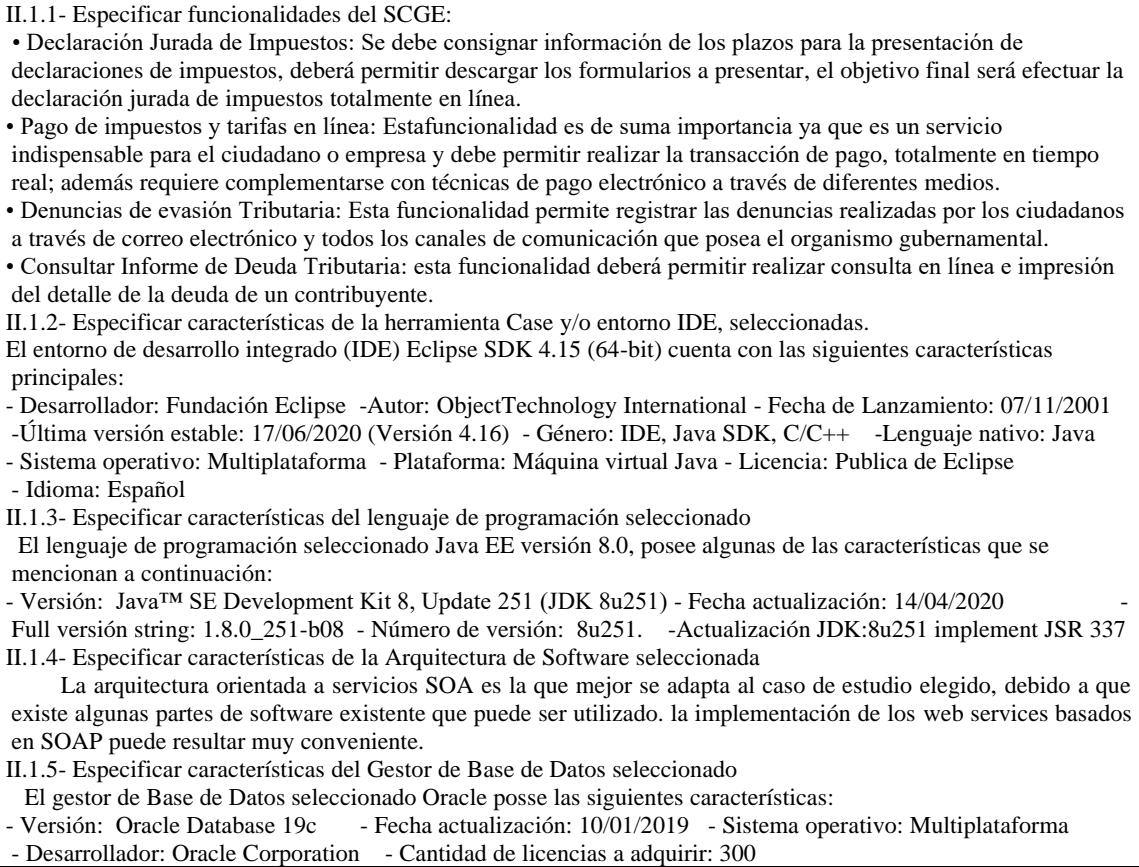 \\
\hline
\end{tabular}

Teniendo en cuenta las actividades previstas para las etapas I y II, a modo de resumen en la figura 4 se puede visualizar el detalle de los ítems seleccionados para los componentes de software, hardware y conectividad. 


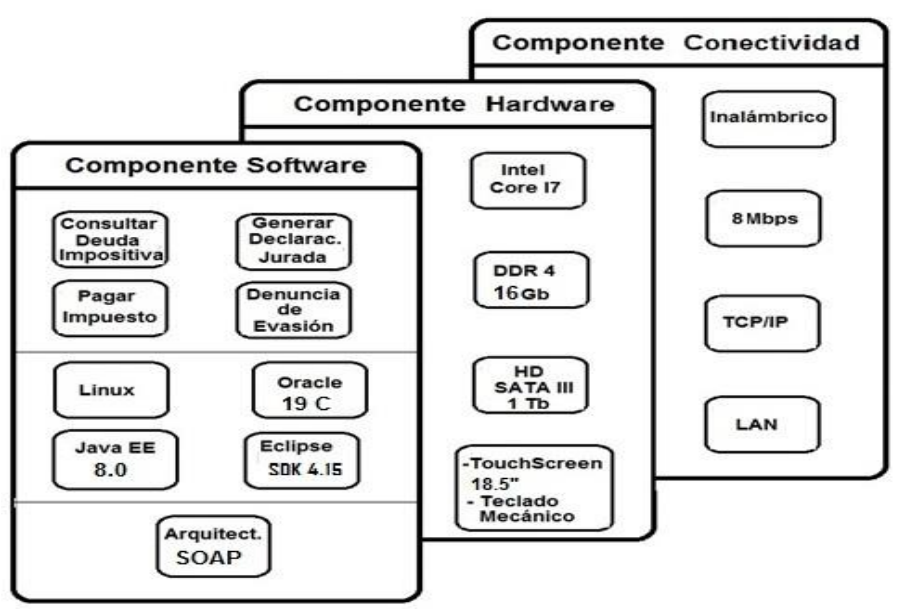

Figura 4. Valores seleccionados y validados para los componentes de software, hardware y conectividad en la etapa I y II

\section{CONCLUSIONES}

Como consecuencia de la investigación desarrollada, se diseñó una metodología que como algo novedoso agrega aspectos relacionados a la estimación de los recursos computacionales, tales como procesamiento, almacenamiento y conectividad, a través de los SC. Es importante señalar que se cumplió con el objetivo propuesto ya que actualmente se está realizando un trabajo experimental que tiene como propósito validar metodo_SCGE, en un caso de estudio. Hasta el momento se validaron las dos primeras etapas y en trabajos futuros se continuarán con el resto de las etapas. El producto final que se obtendrá es un conjunto de especificaciones referidas a los servicios computacionales que se deben implementar, los procesos, el equipamiento y la conectividad necesaria para una solución de gobierno electrónico.

\section{REFERENCIAS}

Boo-04 Booch, G., Rumbaugh, J., Jacobson, I.2004. El Lenguaje Unificado de Modelado. Addison Wesley.

Cas-09 Castro, M., 2009, Análisis de las propiedades y atributos propios de sitios de gobierno electrónico. Tesis de Maestría en Ingeniería de Software. Universidad Nacional de San Luis. Argentina.

Cas-18 Castro, M, C. F. Pons, R. Rodriguez. Propuesta de metodología para el proceso de gobierno electrónico utilizando servicios computacionales. Conisoft 2018. San Luis Potosí. Mexico.

Erl-08 Erl,T.: 2008, SOA Principles of Service Design. Prentice Hall.

Hre-11 Hreño, J.. Bednár, P., Furdik, K., Sabol, T.. 2011. Integration of Government Services using Semantic Technologies. Journal of theoretical and applied electronic commerce research. Res. vol.6 no.1. Disponible en http://www.scielo.cl/scielo.php?script=sci_arttext\&pid=S0718-18762011000100010 .

Mel-04 Mellor, S., Scott, K., Uhl, A., Weise, D. 2004. MDA Distilled, Principles of Model Driven Architecture, Addison-Wesle<y. Professional. 